\title{
Potential application of rLc36 protein for diagnosis of canine visceral leishmaniasis
}

\author{
Camila Tita Nogueira1, Mayara Lúcia Del Cistia², Ana Carolina Urbaczek², Márcia MG Jusi, \\ Angela Maria Arenas Velásquez ${ }^{1}$, Rosângela Zacarias Machado ${ }^{4}$, Henrique Ferreira ${ }^{5}$, \\ Flávio Henrique-Silva ${ }^{6}$, Hélio Langoni ${ }^{7}$, Paulo Inácio da Costa ${ }^{2}$, Márcia AS Graminha ${ }^{1,2}{ }^{+}$ \\ ${ }^{1}$ Universidade Estadual Paulista, Instituto de Química, Campus de Araraquara, Araraquara, SP, Brasil \\ ${ }^{2}$ Universidade Estadual Paulista, Faculdade de Ciências Farmacêuticas, Campus de Araraquara, Araraquara, SP, Brasil \\ ${ }^{3}$ Universidade de São Paulo, Instituto de Química, Campus de São Carlos, São Carlos, SP, Brasil \\ ${ }^{4}$ Universidade Estadual Paulista, Faculdade de Ciências Agrárias e Veterinárias, Campus de Jaboticabal, Jaboticabal, SP, Brasil \\ ${ }^{5}$ Universidade Estadual Paulista, Instituto de Biociências, Campus de Rio Claro, Rio Claro, SP, Brasil \\ ${ }^{6}$ Universidade Federal de São Carlos, Departamento de Genética e Evolução, São Carlos, SP, Brasil \\ ${ }^{7}$ Universidade Estadual Paulista, Faculdade de Medicina Veterinária e de Zootecnia, Campus de Botucatu, Botucatu, SP, Brasil
}

Visceral leishmaniasis (VL) is fatal if left untreated. Infected dogs are important reservoirs of the disease, and thus specific identification of infected animals is very important. Several diagnostic tests have been developed for canine VL (CVL); however, these tests show varied specificity and sensitivity. The present study describes the recombinant protein rLc36, expressed by Leishmania infantum, as potential antigen for more sensitive and specific diagnosis of CVL based on an immunoenzymatic assay. The concentration of $1.0 \mu \mathrm{g} / \mathrm{mL}$ of $\mathrm{rLc} 36$ enabled differentiation of positive and negative sera and showed a sensitivity of $85 \%$ and specificity of $71 \%$ (with $95 \%$ confidence), with an accuracy of $76 \%$.

Key words: canine visceral leishmaniasis - immunodiagnosis - recombinant protein

Kala azar, or visceral leishmaniasis (VL), is a zoonosis caused by protozoan parasites of the Leishmania donovani complex. $L$. donovani and $L$. infantum are the etiological agents of VL in the Old World and L. infantum in the Americas (Ikonomopoulos et al. 2003). In Latin America, VL is transmitted by the bite of infected sand flies Lutzomyia longipalpis (Akhoundi et al. 2016), with domestic dogs (Canis familiaris) as the main reservoir for leishmaniasis infection. Thus, one of the most important approaches for controlling the incidence of human VL is to identify infected dogs (Lira et al. 2006).

Clinical diagnosis of canine VL (CVL) is difficult to achieve because of the nonspecific symptoms in common with other diseases (Mondal et al. 2010). Parasitological methods are based on biopsy or aspiration specimens, but these methods are invasive and present variable sensitivity (Silva et al. 2014).

Among the different serological tests available, enzyme-linked immunosorbent assay (ELISA), indirect immunofluorescence, direct agglutination test, dot-ELISA, and western blotting are the most widely used (Silva et al. 2014). Several Leishmania antigens have been evaluated for serodiagnosis (Badaró et al. 1996, Jensen et al. 1999, Akhoundi et al. 2013), with variable sensitivity and specificity.

doi: 10.1590/0074-02760170171

Financial support: FAPESP (grant \#2010/26732-2), CAPES, PADC.

CTN was supported by the FAPESP fellowship (\#2011/06995-9) and MLDC

by the FAPESP (\#2011/15508-4) and CAPES fellowships.

CTN and MLDC contributed equally to the study.

+ Corresponding author: graminha@fcfar.unesp.br

Received 27 April 2017

Accepted 24 October 2017
Here, we characterised the rLc36 protein encoded by the L. infantum gene LinJ.36.4190 as a potential antigen for developing a more sensitive and specific test for CVL based on ELISA.

Lc36 (GenBank: LinJ.36.4190) was identified through bioinformatic analyses using the EupathDB (eupathdb. org) and TriTrypDB (tritrypdb.org) databases. B-Cell epitope prediction was performed using web server-based software (Saha \& Raghava 2006), and the B-cell epitope database Bcipep (Saha et al. 2005; available from: http:/ www.imtech.res.in/raghava/bcipep). Hydropathicity and the presence of transmembrane domains were also analysed (Kyte \& Doolittle 1982, Cserzo et al. 2004).

L. infantum Lc36 codes for a protein of 733 residues that does not contain transmembrane domains (Supplementary data, Fig. 3). Its product is similar to the proteins of L. donovani and Leishmania mexicana; however, when compared to Leishmania braziliensis, Lc36 shows sequence conservation only at the DNA level, indicating that $L c 36$ is a pseudogene in L. braziliensis (Supplementary data, Fig. 1A-B). Lc36 did not present any sequence similarity when searched against other genome databases including those of Crithidia, Leptomonas, Endotrypanum, and Trypanosoma.

Quantitative polymerase chain reaction (PCR) assays were performed to assess the gene expression levels in different parasite life-cycle stages. For both quantitative and expression assays, we used L. infantum strain MHOM/ BR/1972/LD, which was donated by the Instituto Oswaldo Cruz (FIOCRUZ, Rio de Janeiro, Brazil). The parasites were cultivated as promastigotes in LIT (Liver Infusion Tryptose - BD Bioscience, San Jose, CA, USA) medium supplemented with $10 \%$ foetal bovine serum at $26^{\circ} \mathrm{C}, \mathrm{pH} 7.2$. 
For quantitative PCR assays, we used mouse peritoneal macrophages collected from adult male Swiss albino mice, infected with $L$. infantum as previously described (Velásquez et al. 2017), except that the assay was performed in $25-\mathrm{cm}^{2}$ cell culture flasks (Nunc ${ }^{\mathrm{TM}}$, Roskilde, Denmark). These assays were approved by the Ethics Committee for Animal Experimentation (Protocol CEUA/FCF/CAr no. 40/2015) in agreement with Sociedade Brasileira de Ciência de Animais de Laboratório and Conselho Nacional de Controle de Experimentação Animal.

For RNA extraction and real-time PCR analysis, primers RT_36_Forward 5'-GTTCGTCACCGTTGTCTTC-3' and RT_36_Reverse 5'-GTCGTG CTTCCTGCTATTC-3' were designed using the software tools GeneRunner (http://www.generunner.com) and Primer Express version 3.0. Total promastigotes and infected and non-infected macrophage RNA were extracted using TRIzol ${ }^{\circledR}$ Reagent (Life Technologies, Carlsbad, CA, USA) according to the manufacturer's protocol. Intracellular amastigote RNA was extracted at 18,48 , and $72 \mathrm{~h}$ post-infection. For cDNA synthesis, we used $1 \mu \mathrm{g}$ of total RNA, the 3'Race System for Rapid Amplification of cDNA ends kit (Life Technologies), and random hexamer primers. Real-time PCR assays were carried out in a StepOnePlus ${ }^{\mathrm{TM}}$ Real-Time PCR System v2.3 (Applied Biosystems, Foster City, CA, USA) with initial denaturation at $95^{\circ} \mathrm{C}$ for $20 \mathrm{~s}$ followed by 40 denaturation cycles at $95^{\circ} \mathrm{C}$ for $15 \mathrm{~s}$, annealing at $60^{\circ} \mathrm{C}$ for $1 \mathrm{~min}$, and extension at $95^{\circ} \mathrm{C}$ for $15 \mathrm{~s}$. For each reaction, 1x Fast Power SYBR ${ }^{\circledR}$ Green Master Mix (Applied Biosystems), $0.1 \mu \mathrm{M}$ of each primer, and $10 \mathrm{ng}$ of each cDNA sample was added in $10-\mu \mathrm{L}$ of PCR mixture. A 10 -fold dilution series of plasmid DNA (recombinant plasmid pET28a+_Lc36) was used for absolute standard curve construction to determine the efficiency. Assays were carried out in triplicate. Absolute amounts of PCR products were determined based on the threshold cycle $(C \mathrm{t})$, by interpolating each $C \mathrm{t}$ value from each sample on the corresponding standard curve and by the size of plasmid DNA template (7588 base pairs) using the equation described in the Supplementary data (Equation 1). For graphics design, we used software Origin ${ }^{\circledR}$ and Excel ${ }^{\circledR}$ software. These assays revealed that the gene is likely expressed in amastigote cells, the form responsible for the disease's clinical manifestations. The absolute standard curve parameters showed an efficiency of $99.23 \%$ and linear regression coefficient $\left(\mathrm{R}^{2}\right)$ of 0.98 (Supplementary data, Table II, Figs 5, 6). Fig. 1 shows that the cDNA from intracellular amastigotes of $18 \mathrm{~h}$ and $48 \mathrm{~h}$ post-infection macrophages had $\mathrm{C}_{\mathrm{T}}$ values of $36.0 \pm 0.8$ and $32.2 \pm 0.6$, respectively, while $C_{T}$ values higher than 40 were observed for cDNA from promastigotes and intracellular amastigotes at $72 \mathrm{~h}$ post-infection. These data were converted to cDNA copy numbers (Supplementary data, Table II, Figs 5-7) and Fig. 1 illustrates that the cDNA copy number was higher in intracellular amastigotes at $48 \mathrm{~h}$ post-infection (20 copies per reaction) compared to in parasites at 18 $\mathrm{h}$ post-infection (1 copy per reaction). Thus, the results suggest that this gene has a stage-specific expression pattern and indicate that $L$. infantum Lc36 is amastigotestage specific and is expressed at $48 \mathrm{~h}$ post-infection.

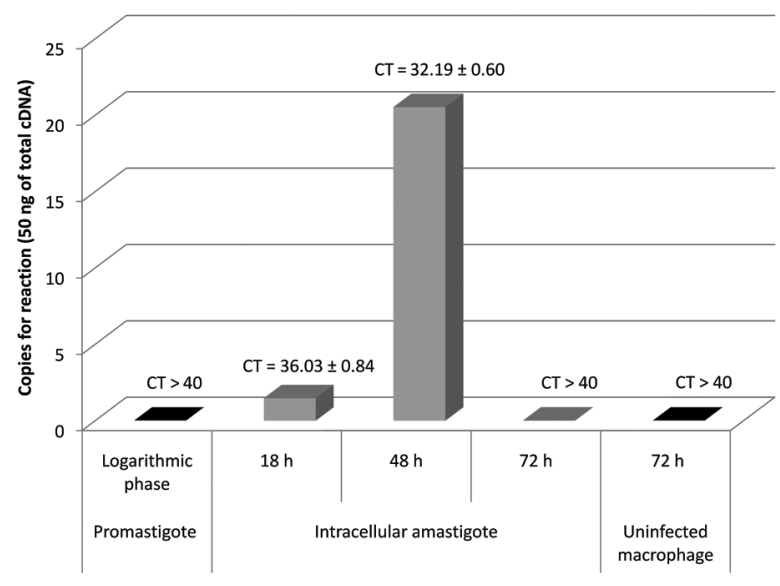

Fig. 1: quantitative analysis of Lc36 gene expression in different lifecycle stage forms of Leishmania infantum. Source: authors.

Leishmania has an intracellular life stage and is likely to encounter reactive oxygen species produced by the macrophage, which can induce DNA damage. Thus, stage-specific amastigote genes may be involved in parasite survival in macrophages (Genois et al. 2014). Interestingly, $L c 36$ codes for a protein of unknown function containing a nucleotidyl transferase domain which is possibly involved in nucleic acid metabolism, including DNA repair, which may play an important role in protecting Leishmania amastigote genome integrity during its exposure to reactive oxygen species produced by macrophages (Vaňáčová et al. 2005). For application and diagnosis, some amastigote stage-specific proteins present antigenic properties recognised by the host, such as the A2 protein, which has been evaluated for VL serodiagnosis and vaccine application (Coelho et al. 2012, Martins et al. 2015). As these data indicated the importance of rLc36 for the development of VL diagnosis assays, we tested the performance in ELISA. We mapped linear B cell epitopes within the Lc36 coding region. The protein region from residues 1 to 255 was selected and evaluated for serodiagnosis of CVL (Supplementary data, Table I, Fig. 2). The chosen fragment containing 765 base pairs was amplified and cloned into pET28a + for heterologous expression in Escherichia coli. For expression and purification of rLc36, a recombinant plasmid named as pET28a_Lc36 was constructed (cloning strategy, in Supplementary data). Protein expression was carried out in a $250-\mathrm{mL}$ bacterial culture in LB medium containing $25 \mu \mathrm{g} / \mathrm{mL}$ kanamycin, at $250 \mathrm{rpm}$ and $37^{\circ} \mathrm{C}$. The expression of rLc36 was induced by $0.5 \mathrm{mM}$ IPTG and growth was continued at $30^{\circ} \mathrm{C}$ for $4 \mathrm{~h}$. Next, a bacterial extract was prepared, and the recombinant protein was purified by affinity chromatography using Poly-Prep Chromatography columns (Bio-Rad, Hercules, CA, USA) with the resin Chelating Sepharose TM Fast Flow (GE Healthcare, Little Chalfont, UK). The recombinant protein was eluted with an imidazole gradient ranging from 20 to $200 \mathrm{mM}$. The $\mathrm{His}_{6}$-tagged fusion protein (rLc36), 
A

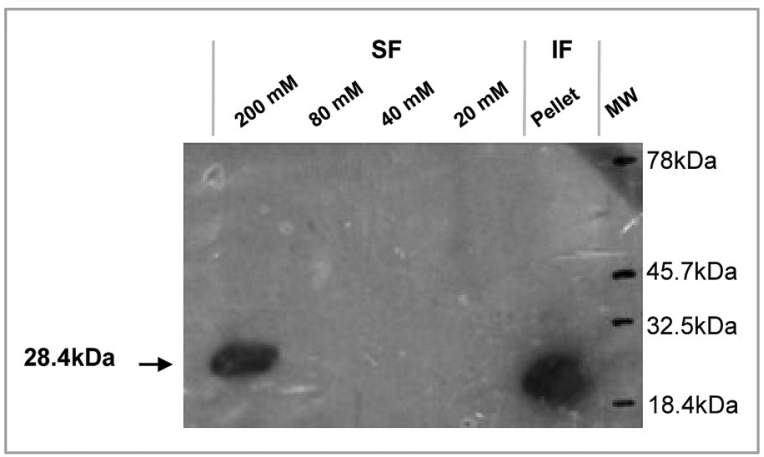

B

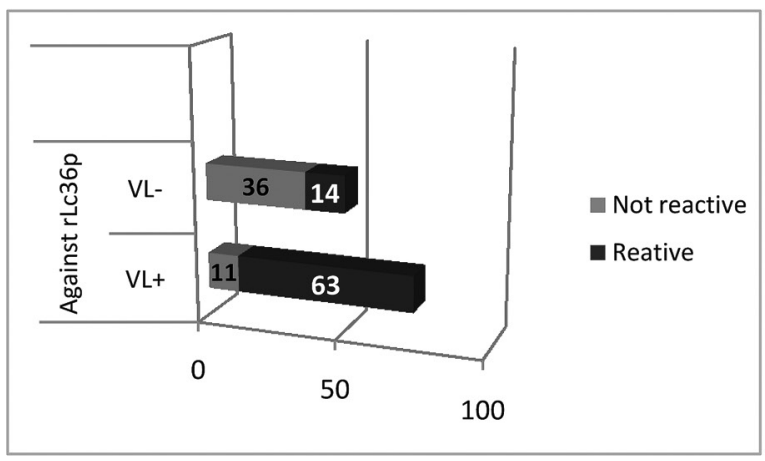

Fig. 2: purification and reactivity of protein rLc36. (A) Western blot analysis of purified His6-tagged rLc36. rLc36 was expressed in Escherichia coli BL21(DE3) after isopropyl $\beta$-d-thiogalactopyranoside induction for $4 \mathrm{~h}$ and purified by nickel-affinity chromatography using a gradient of imidazole $(20,40,80$, and $200 \mathrm{mM})$. The purified recombinant protein was assessed by western blotting using anti-histidine mouse antibody. Non-soluble fraction (IF) obtained after cell lysis and before column purification step. Protein molecular weight determined by Kaleidoscope. (B) Reactivity of antigens rLc36. The cut-off used was 0.191 , for a confidence of $95 \%$, using 20 negative sera for visceral leishmaniasis. Source: authors.

presenting the expected molecular weight, was successfully purified (Supplementary data, Fig. 4).

rLc36 was subjected to $12 \%$ SDS-PAGE and transferred to nitrocellulose membranes. Membranes were blocked with 5\% non-fat dry milk in $1 \mathrm{x}$ PBS, pH 7.3, and incubated overnight at $4^{\circ} \mathrm{C}$. The membrane was washed with $0.05 \%$ Tween 20 in PBS and incubated for $2 \mathrm{~h}$ in the presence of monoclonal anti-polyhistidine 1:1500 (Sigma, St. Louis, MO, USA). After washing, the membrane was treated for $1 \mathrm{~h}$ with peroxidase-conjugated antimouse secondary antibody (1:40,000), and subsequently developed successively with luminol [50 mL Tris- $\mathrm{HCl} 1$ $\mathrm{M} \mathrm{pH} \mathrm{8.5,} \mathrm{in} \mathrm{H}_{2} \mathrm{O}, 5 \mathrm{~mL}$ of solution $\mathrm{A}(0.22 \mathrm{~g}$ luminol in $5 \mathrm{~mL}$ DMSO), $2.2 \mathrm{~mL}$ of solution $\mathrm{B}(0.033 \mathrm{~g}$ of $\mathrm{p}$ coumaric acid, $2.2 \mathrm{~mL}$ of DMSO)], and $0.3 \% \mathrm{H}_{2} \mathrm{O}_{2}$. The membranes were placed in contact with a photographic film for $5 \mathrm{~min}$ and bands were visualised by the addition of Step ${ }^{\mathrm{TM}}$ NBT/BCIP (Pierce, Rockford, IL, USA). Western blotting analyses revealed the presence of the purified $\mathrm{His}_{6}$-tagged protein in the $200 \mathrm{mM}$ imidazole-eluate soluble fraction and in the inclusion bodies (Fig. 2A).

For ELISA, 96-well plates were coated with the recombinant protein $\mathrm{rLc} 36$ in $100 \mu \mathrm{L}$ of coating buffer for $18 \mathrm{~h}$ at $4^{\circ} \mathrm{C}$. For this assay, $1 \mu \mathrm{g} / \mathrm{mL}$ of $\mathrm{rLc} 36$ was used, based on a titration curve developed to determine the optimal recombinant protein concentration. VL-positive sera used in this study were obtained from the area of Campo Grande, Minas Gerais, Brazil, in which CVL is endemic, whereas negative sera were obtained from Jaboticabal, São Paulo, Brazil, a non-endemic area. The sera were selected for CVL based on Leishmania chagasi-specific total IgG and L. chagasi-specific IgG subclass ELISAs with a confidence index of 95\%. Both sera groups were confirmed by direct parasitological methods and indirect immunofluorescence. In the ELISA for rLc36, the plates were blocked with $2 \%$ non-fat dry milk solution for $1 \mathrm{~h}$ at $37^{\circ} \mathrm{C}$. After three washes with PBS Tween 80 at $0.05 \%$, the plates were incubated with $100 \mu \mathrm{L}$ of canine sera for $1 \mathrm{~h}$ at $37^{\circ} \mathrm{C}$. Sera samples were diluted 1:200 in PBS with 2\% normal rabbit serum. Plates were then washed three times with PBS Tween 80 and incubated with 1:4000 of alkaline phosphatase conjugated to anti-dog IgG (Sigma) for $1 \mathrm{~h}$ at $37^{\circ} \mathrm{C}$. Plates were washed and $100 \mu \mathrm{L}$ diethanolamine solution $(\mathrm{pH}$ 9.8) containing a substrate for phosphatase (4-nitrophenyl phosphate disodium salt hexahydrate, Sigma) was added to each well. The optical density was measured at $405 \mathrm{~nm}$ in an ELISA microplate spectrophotometer (Robonik ${ }^{\circledR}$ - Readwell Touch Automatic ELISA Plate Analyser). Sensitivity and specificity values were determined by discriminating the absorbance data of sera true-positives and true-negatives, as well as false-positives and false-negatives, using cut-off method analysis described by Frey et al. (1998). The accuracy predictive value was also determined (Kawamura 2002). For a complete description, see Supplementary data (Equations 2, 3, 4). The $\mathrm{His}_{6}$-tagged protein, confirmed as rLc36, was analysed for antigenic potential against canine sera; 74 sera were previously demonstrated to be positive for $\mathrm{CVL}$ while another 50 were negative for this disease (Fig. 2B). Sensitivity and specificity of the rLc36 against the CVL sera were $85 \%$ and $72 \%$, respectively, with a cut-off with $95 \%$ confidence. Another group of 39 sera samples was separated in symptomatic $(\mathrm{n}=33)$ and asymptomatic $(\mathrm{n}$ $=6$ ) subgroups and tested with a cut-off with $99 \%$ confidence (Fig. 3). All symptomatic sera were confirmed to be positive for CVL, while for asymptomatic sera, the ELISA based on rLC36 detected four of six asymptomatic samples (Supplementary data, Table V).

Several recombinant proteins have shown variable effectiveness for leishmaniasis serodiagnosis (Badaró et al. 1996, Bhatia et al. 1999, Jensen et al. 1999, Raj et al. 1999) and others present high amino acid sequence homologies to human and/or canine counterparts, interfering with the applicability of the tests (Maalej et al. 2003, Coelho et al. 2012, Celeste et al. 2014). Thus, based on BLAST analyses revealing a lack of homology to other parasitic sequences, rLc36 may be useful for developing a new and specific diagnostic test for CVL. 


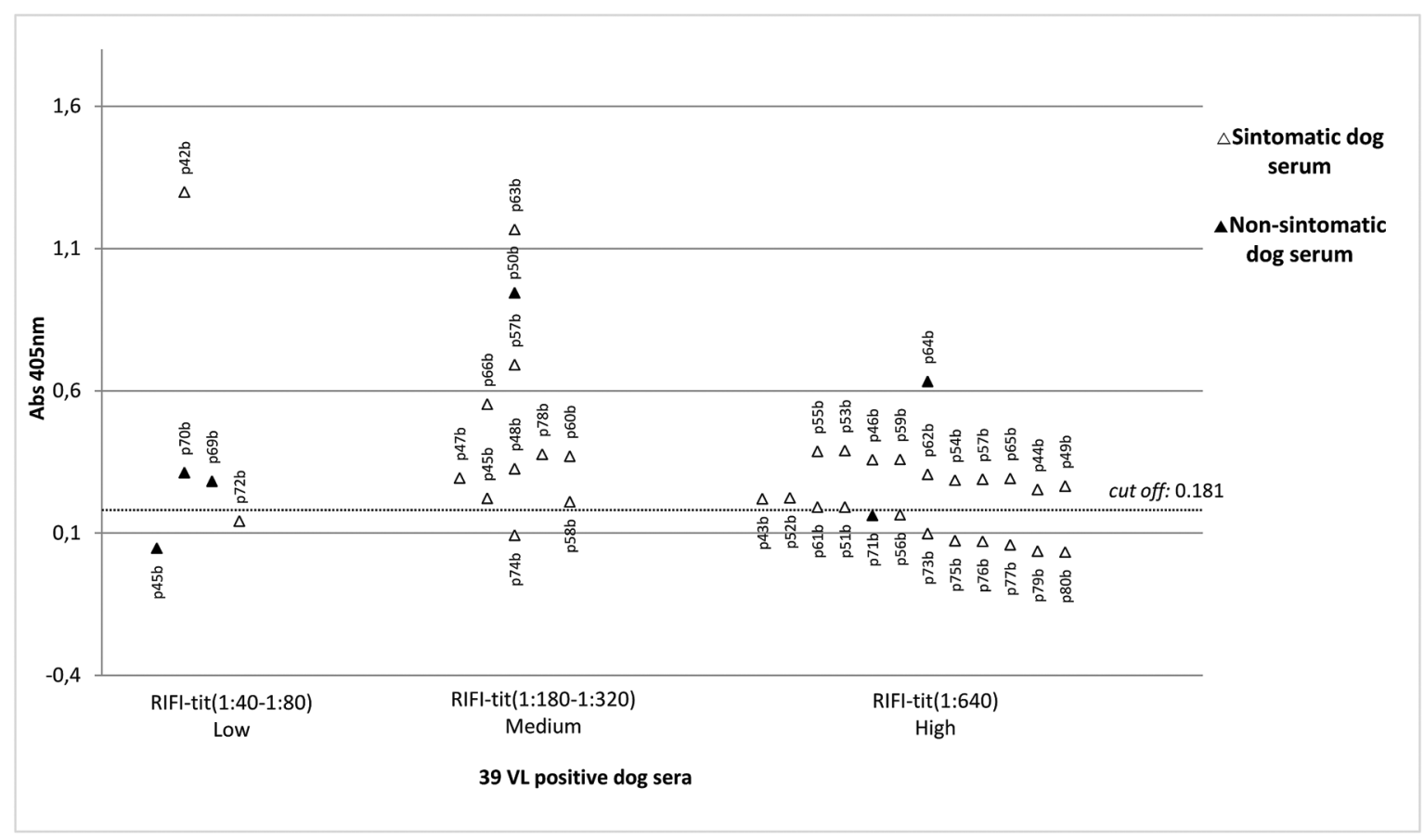

Fig. 3: reactivity of 39 positive sera for canine visceral leishmaniasis (CVL) versus rLc36. The dotted line refers to a cut-off of 0.181 , calculated based on a confidence of $99 \%$, using 20 negative sera for VL. Source: authors.

In conclusion, the rLc36 protein, which is likely expressed in amastigote forms of L. infantum, was capable of differentiating positive from negative CVL sera and showed a sensitivity and specificity of $85 \%$ and $71 \%$, respectively, with a confidence of $95 \%$ and accuracy of $76 \%$, in a randomly chosen population. The protein was also able to identify asymptomatic animals. These results indicate that $\mathrm{rLc} 36$ is promising for CVL serodiagnosis.

\section{ACKNOWLEDGEMENTS}

To professor Paul A Michels for critically reviewing the manuscript, and Wanderson HC Oliveira for the English revision.

\section{AUTHORS' CONTRIBUTION}

CTN and MLDC provided the experimental data, wrote and reviewed the full manuscript; MASG and PIC designed and reviewed the full manuscript; ACU, MMGJ, AMAV, RZM, HF, FHS and HL provided assistance with experimental data.

\section{REFERENCES}

Akhoundi B, Mohebali, M, Shojaee S, Jalali M, Kazemi B, Bandehpour M, et al. Rapid detection of human and canine visceral leishmaniasis: assessment of a latex agglutination test based on the A2 antigen from amastigote forms of Leishmania infantum. Exp Parasitol. 2013; 133(3): 307-13.

Akhoundi M, Kuhls K, Cannet A, Votýpka J, Marty P, Delaunay P, et al. A historical overview of the classification, evolution, and dispersion of leishmania parasites and sandflies. PLoS Negl Trop Dis. 2016; 10(3): e0004349.

Badaró R, Benson D, Eulálio MC, Freire M, Cunha S, Netto EM, et al. rK39: a cloned antigen of Leishmania chagasi that predicts active visceral leishmaniasis. J Infect Dis. 1996; 173(3): 758-61.
Bhatia A, Daifalla NS, Jen S, Badaro R, Reed SG, Skeiky YAW. Cloning, characterization and serological evaluation of K9 and K26: two related hydrophilic antigens of Leishmania chagasi. Mol Biochem Parasitol. 1999; 102(2): 249-61.

Celeste BJ, Sánchez MC, Ramos-Sánchez EM, Castro LG, Costa FA, Goto H. Recombinant Leishmania infantum heat shock protein 83 for the serodiagnosis of cutaneous, mucosal, and visceral leishmaniases. Am J Trop Med Hyg. 2014; 90(5): 860-5.

Coelho VTS, Oliveira JS, Valadares DG, Chávez-Fumagalli MA, Duarte MC, Lage PS, et al. Identification of proteins in promastigote and amastigote-like Leishmania using an immunoproteomic approach. PLoS Negl Trop Dis. 2012; 6(1): e1430.

Cserzo M, Eisenhaber F, Eisenhaber B, Simon I. TM or not TM: transmembrane protein prediction with low false positive rate using DAS-TMfilter. Bioinformatics. 2004; 20(1): 136-7.

Frey A, Di Canzio J, Zurakowski D. A statistically defined endpoint titer determination method for immunoassays. J Immunol Methods. 1998; 221(1-2): 35-41.

Genois MM, Paquet ER, Laffitte MC, Maity R, Rodrigue A, Ouellette $\mathrm{M}$, et al. DNA repair pathways in trypanosomatids: from DNA repair to drug resistance. Microbiol Mol Biol Rev. 2014; 78(1): 40-73.

Ikonomopoulos J, Kokotas S, Gazouli M, Zavras A, Stoitsiou M, Gorgoulis VG. Molecular diagnosis of leishmaniosis in dogs: comparative application of traditional diagnostic methods and the proposed assay on clinical samples. Vet Parasitol. 2003; 113(2): 99-113.

Jensen ATR, Gasim S, Moller T, Ismail A, Gaafar A, Kemp M, et al. Serodiagnosis of Leishmania donovani infections: assessment of enzyme-linked immunosorbent assays using recombinant $L$. donovani gene B protein (GBP) and a peptide sequence of $L$. donovani GBP. Trans R Soc Trop Med Hyg. 1999; 93(2): 157-60.

Kawamura T. Interpretação de um teste sob a visão epidemiológica. Eficiência de um teste. Arq Bras Cardiol. 2002; 79(4): 437-41. 
Kyte J, Doolittle RF. A simple method for displaying the hydropathic character of a protein. J Mol Biol. 1982; 157(1): 105-32.

Larsen JEP, Lund O, Nielsen M. Improved method for predicting linear B-cell epitopes. Immunome Res. 2006; 2: 2.

Lira RA, Cavalcanti MP, Nakazawa M, Ferreira AGP, Silva ED, Abath FGC, et al. Canine visceral leishmaniosis: a comparative analysis of the EIE-leishmaniose-visceral-canina-Bio-Manguinhos and the IFI-leishmaniose-visceral-canina-Bio-Manguinhos kits. Vet Parasitol. 2006; 137(1-2): 11-6.

Maalej IA, Chenik M, Louzir H, Ben Salah A, Bahloul C, Amri F, et al. Comparative evaluation of ELISAs based on ten recombinant or purified Leishmania antigens for the serodiagnosis of Mediterranean visceral leishmaniasis. Am J Trop Med Hyg. 2003; 68(3): 312-20.

Martins VT, Chávez-Fumagalli MA, Lage DP, Duarte MC, Garde E, Costa LE, et al. Antigenicity, immunogenicity and protective efficacy of three proteins expressed in the promastigote and amastigote stages of Leishmania infantum against visceral leishmaniasis. PLoS ONE. 2015; 10(10): e0141496.

Mondal S, Bhattacharya P, Ali N. Current diagnosis and treatment of visceral leishmaniasis. Expert Rev Anti Infect Ther. 2010: 8(8): 919-44.
Raj VS, Ghosh A, Dole VS, Madhubala R, Myler PJ, Stuart KD. Serodiagnosis of leishmaniasis with recombinant ORFF antigen. Am J Trop Med Hyg. 1999; 61(3): 482-7.

Saha S, Bhasin M, Raghava GPS. Bcipep: a database of B-cell epitopes. BMC Genomics. 2005; 6: 79.

Saha S, Raghava GP. Prediticion of continuous B-cell epitopes in an antigen using recurrent neural network. Proteins. 2006; 65(1): 40-8.

Silva DT, Starke-Buzetti WA, Alves-Martin MF, Paixão MS, Tenório MS, Lopes ML. Comparative evaluation of several methods for canine visceral leishmaniasis diagnosis. Rev Bras Parasitol Vet. 2014; 23(2): 179-86.

Vaňáčová Š, Wolf J, Martin G, Blank D, Dettwiler S, Friedlein A, et al. A new yeast poly(A) polymerase complex involved in RNA quality control. PLoS Biol. 2005; 3(6): 986-97.

Velásquez AMA, Ribeiro WC, Venn V, Castelli S, Camargo MS, de Assis RP, et al. Efficacy of a binuclear cyclopalladated compound therapy for cutaneous leishmaniasis in the murine model of infection with Leishmania amazonensis and its inhibitory effect on topoisomerase 1B. Antimicrob Agents Chemother. 2017; 61(8): pii: e00688-17. 